\title{
Special Issue on Novel Insights into Orbital Angular Momentum Beams: From Fundamentals, Devices to Applications
}

\author{
Yang Yue ${ }^{1, *}$, Hao Huang ${ }^{2}$, Yongxiong Ren ${ }^{2}$, Zhongqi Pan ${ }^{3}$ and Alan E. Willner ${ }^{2}$ \\ 1 Institute of Modern Optics, Nankai University, Tianjin 300350, China \\ 2 Department of Electrical Engineering, University of Southern California, Los Angeles, CA 90089, USA \\ 3 Department of Electrical and Computer Engineering, University of Louisiana at Lafayette, \\ Lafayette, LA 70504, USA \\ * Correspondence: yueyang@nankai.edu.cn
}

Received: 13 June 2019; Accepted: 24 June 2019; Published: 27 June 2019

\section{Introduction}

It is well-known now that angular momentum carried by elementary particles can be categorized as spin angular momentum (SAM) and orbital angular momentum (OAM). In the early 1900s, Poynting recognized that a particle, such as a photon, can carry SAM, which has only two possible states, i.e., clockwise and anticlockwise circular polarization states. However, only fairly recently, in 1992, Allen et al. discovered that photons with helical phase fronts can carry OAM, which has infinite orthogonal states [1]. In the past two decades, the OAM-carrying beam, due to its unique features, has gained increasing interest from many different research communities, including physics, chemistry and engineering [2,3]. Its twisted phase front and intensity distribution have enabled a variety of applications, such as micromanipulation [4-6], laser beam machining [7-9], nonlinear matter interactions [10-12], imaging [13-15], sensing [16,17], quantum cryptography, and classical communications [18-23].

\section{Special Issue Papers}

This special issue aims to explore the novel insights of OAM beams. It focuses on state-of-the-art advances in fundamental theories, devices, and applications as well as future perspectives of OAM beams. The collected papers have well accomplished these goals by contributing leading-edge derivation, analysis, and experiments with significant results. The topics cover OAM generation and reception, multiplexing and de-multiplexing, device and system. The frequencies range from radio frequency $(\mathrm{RF})$ to infrared wave, while the techniques behind extend from integrated photonics, fiber optics, free-space optics, to dielectric. The special issue consists of three review papers, one communication and five research articles.

More specifically, from the physical perspective, Prof. Barnett and his group have a review paper on the helicity of light, and how it can be both produced and used in light-matter interactions [24]. The paper starts from the form of the helicity density and its associated continuity equation in free space, in the presence of local currents and charges, and upon interaction with bulk media, leading to the characterization of both microscopic and macroscopic sources of helicity.

Regarding OAM beam generation technologies, Prof. Liu's group reviews the generation of OAM modes using fiber systems [25]. This review paper first introduces the basic concepts of fiber modes and the generation and detection theories of OAM modes. Next, fiber systems based on different devices are introduced, including long-period fiber grating, mode-selective coupler, microstructured 
optical fiber, and the photonic lantern. Finally, the key challenges and prospects for fiber OAM mode systems are discussed.

Another review paper, focusing on tunable OAM generation, is contributed from Prof. Wu's group [26]. The authors classify the tunable OAM mode generation methods into three categories, according to the OAM and polarization states. The fiber-based and free-space generation methods are categorized into three types according to the controllable variables, respectively. Last, the pros and cons of each generation method are analyzed and the key challenges for tunable OAM modes are discussed.

Most fiber-based or free-space OAM beam generators are bulky, slow, and cannot withstand high powers. Prof. Litchinitser's group design and experimentally demonstrate an ultra-fast, compact chalcogenide-based all-dielectric metasurface beam converter, which has the ability to transform a Hermite-Gaussian (HG) beam into an OAM beam at near infrared wavelength [27]. The topological charge carried by the output OAM beam can be switched between positive and negative values, and the device provides high transmission efficiency.

For the reception of OAM radio waves, Dr. Klemes contributes a communication article using pseudo-Doppler interpolation techniques [28]. The method can be used to receive OAM waves in the far field of an antenna transmitting multiple OAM modes, each carrying a separate data stream at the same RF. The frequency domain method provides a higher signal-to-noise ratio (SNR) than using spatial-domain OAM reception techniques. Moreover, no more than two receiving antennas are necessary to separate any number of OAM modes in principle.

In OAM communications systems, different OAM beams can carry multiple data channels, boosting the spectral efficiency and capacity significantly. Consequently, the simultaneous processing of OAM beams is necessary, and OAM multiplexing/de-multiplexing devices are key enablers of such systems. Prof. Li et al. contribute an article on mode-selective photonic lanterns for OAM mode division multiplexing [29]. The authors design a three-mode OAM mode-selective photonic lantern by optimizing the taper length with small mode crosstalk, which employs only a single mode fiber port to selectively generate each OAM mode.

In a more integrated manner, Prof. Romanato and his group explore holographic Silicon metasurfaces for OAM de-multiplexing based on OAM-mode projection [30]. The device uses Pancharatnam-Berry optical elements (PBOEs) and can de-multiplex beams with different polarization and OAM states at the wavelength of $1310 \mathrm{~nm}$. The geometric-phase control is achieved by inducing a spatially-dependent form-birefringence on a silicon substrate, patterned with properly-oriented subwavelength gratings.

There are two experimental demonstrations for free-space OAM communications systems, one in the $1550 \mathrm{~nm}$ optical regime, and the other on $28 \mathrm{GHz}$ RF frequency band. Dr. Qu and Prof. Djordjevic investigate turbulence mitigation methods in free-space optical OAM communications system based on coded modulation [31]. Adaptive optics, channel coding, Huffman coding combined with low-density parity-check (LDPC) coding, and spatial offset are used for turbulence mitigation, achieving a total data transmission capacity of 500 Gbps.

Finally, Dr. Lee and colleagues evaluate the performance of OAM-based wireless communications systems [32]. To overcome the beam divergence of OAM multiplexing, the authors use a combination of multi-input multi-output (MIMO) and OAM technology, achieving a new milestone in point-to-point transmission rates at $100 \mathrm{Gbps}$ for a $10 \mathrm{~m}$ transmission distance.

\section{Perspectives}

It has just been 27 years since the discovery of OAM by Les Allen and his co-workers. Within this fairly short period of time, an extensive research community has been established globally, and OAM theories have been further improved. Especially during the past decade, OAM related devices and applications have experienced significant growth. From this trend, we are expecting the OAM field to 
continue grow with novel and unique applications to debut one after another. Hopefully, more OAM related technologies can be commercialized in the near future to enable new industry and serve society.

Acknowledgments: The guest editors would like to thank all the authors for submitting their excellent work to this special issue. Furthermore, we would like to thank all the reviewers for their outstanding job in evaluating the manuscripts and providing helpful comments. The guest editors also would like to thank the MDPI team involved in the preparation, editing, and managing of this special issue. Finally, we would like to express our sincere gratitude to Ms. Lucia Li, the contact editor of this special issue, for her kind, efficient, professional guidance and support through the whole process. We would not be able to reach the above collection of high quality papers without this joint effort.

Conflicts of Interest: The authors declare no conflict of interest.

\section{References}

1. Allen, L.; Beijersbergen, M.W.; Spreeuw, R.J.C.; Woerdman, J.P. Orbital angular momentum of light and the transformation of Laguerre-Gaussian laser modes. Phys. Rev. A 1992, 45, 8185-8189. [CrossRef] [PubMed]

2. Yao, A.M.; Padgett, M.J. Orbital angular momentum: Origins, behavior and applications. Adv. Opt. Photon. 2011, 3, 161-204. [CrossRef]

3. Padgett, M.J. Orbital angular momentum 25 years on [Invited]. Opt. Express 2017, 25, 11265-11274. [CrossRef] [PubMed]

4. Friese, M.E.J.; Nieminen, T.A.; Heckenberg, N.R.; Rubinsztein-Dunlop, H. Optical alignment and spinning of laser-trapped microscopic particles. Nature 1998, 394, 348-350. [CrossRef]

5. Dholakia, K.; Cizmar, T. Shaping the future of manipulation. Nat. Photonics 2011, 5, 335-342. [CrossRef]

6. Padgett, M.; Bowman, R. Tweezers with a twist. Nat. Photonics 2011, 5, 343-348. [CrossRef]

7. Friese, M.; Rubinsztein-Dunlop, H.; Gold, J.; Hagberg, P.; Hanstorp, D. Optically driven micromachine elements. Appl. Phys. Lett. 2001, 78, 547-549. [CrossRef]

8. Knoner, G.; Parkin, S.; Nieminen, T.A.; Loke, V.L.Y.; Heckenberg, N.R.; Rubinsztein-Dunlop, H. Integrated optomechanical microelements. Opt. Express 2007, 15, 5521-5530. [CrossRef]

9. Ladavac, K.; Grier, D. Micro-optomechanical pumps assembled and driven by holographic optical vortex arrays. Opt. Express 2004, 12, 1144-1149. [CrossRef]

10. Firth, W.; Skryabin, D. Optical solitons carrying orbital angular momentum. Phys. Rev. Lett. 1997, 79, 2450-2453. [CrossRef]

11. Litchinitser, N.M. Applied physics. Structured light meets structured matter. Science 2012, 337, $1054-1055$. [CrossRef] [PubMed]

12. Gauthier, D.; Ribic, P.R.; Adhikary, G.; Camper, A.; Chappuis, C.; Cucini, R.; DiMauro, L.F.; Dovillaire, G.; Frassetto, F.; Géneaux, R.; et al. Tunable orbital angular momentum in high-harmonic generation. Nat. Commun. 2017, 8, 14971. [CrossRef]

13. Hell, S.W.; Wichmann, J. Breaking the diffraction resolution limit by stimulated emission: Stimulated-emission-depletion fluorescence microscopy. Opt. Lett. 1994, 19, 780-782. [CrossRef]

14. Swartzlander, G.A.; Ford, E.L.; Abdul-Malik, R.S.; Close, L.M.; Peters, M.A.; Palacios, D.M.; Wilson, D.W. Astronomical demonstration of an optical vortex coronagraph. Opt. Express 2008, 16, 10200-10207. [CrossRef] [PubMed]

15. Jesacher, A.; Ritsch-Marte, M.; Piestun, R. Three-dimensional information from two-dimensional scans: A scanning microscope with postacquisition refocusing capability. Optica 2015, 2, 210-213. [CrossRef]

16. Cvijetic, N.; Milione, G.; Ip, E.; Wang, T. Detecting lateral motion using light's orbital angular momentum. Sci. Rep. 2015, 5, 15422. [CrossRef] [PubMed]

17. Xie, G.; Song, H.; Zhao, Z.; Milione, G.; Ren, Y.; Liu, C.; Zhang, R.; Bao, C.; Li, L.; Wang, Z.; et al. Using a complex optical orbital-angular-momentum spectrum to measure object parameters. Opt. Lett. 2017, 42, 4482-4485. [CrossRef] [PubMed]

18. Wang, J.; Yang, J.Y.; Fazal, I.M.; Ahmed, N.; Yan, Y.; Huang, H.; Ren, Y.X.; Yue, Y.; Dolinar, S.; Tur, M.; et al. Terabit free-space data transmission employing orbital angular momentum multiplexing. Nat. Photonics 2012, 6, 488-496. [CrossRef]

19. Bozinovic, N.; Yue, Y.; Ren, Y.; Tur, M.; Kristensen, P.; Huang, H.; Willner, A.E.; Ramachandran, S. Terabit-scale orbital angular momentum mode division multiplexing in fibers. Science 2013, 340, 1545-1548. [CrossRef] 
20. Krenn, M.; Handsteiner, J.; Fink, M.; Fickler, R.; Ursin, R.; Malik, M.; Zeilinger, A. Twisted light transmission over 143 km. Proc. Natl. Acad. Sci. USA 2013, 113, 13648-13653. [CrossRef]

21. Yan, Y.; Xie, G.; Lavery, M.P.J.; Huang, H.; Ahmed, N.; Bao, C.; Ren, Y.; Cao, Y.; Li, L.; Zhao, Z.; et al. High-capacity millimetre-wave communications with orbital angular momentum multiplexing. Nat. Commun. 2014, 5, 4876. [CrossRef] [PubMed]

22. Vallone, G.; D’Ambrosio, V.; Sponselli, A.; Slussarenko, S.; Marrucci, L.; Sciarrino, F.; Villoresi, P. Free-space quantum key distribution by rotation-invariant twisted photons. Phys. Rev. Lett. 2014, 113, 060503. [CrossRef] [PubMed]

23. Sit, A.; Bouchard, F.; Fickler, R.; Gagnon-Bischoff, J.; Larocque, H.; Heshami, K.; Elser, D.; Peuntinger, C.; Günthner, K.; Heim, B.; et al. High-dimensional intracity quantum cryptography with structured photons. Optica 2017, 4, 1006-1010. [CrossRef]

24. Crimin, F.; Mackinnon, N.; Götte, J.B.; Barnett, S.M. Optical helicity and chirality: Conservation and sources. Appl. Sci. 2019, 9, 828. [CrossRef]

25. Zhang, H.; Mao, B.; Han, Y.; Wang, Z.; Yue, Y.; Liu, Y. Generation of orbital angular momentum modes using fiber systems. Appl. Sci. 2019, 9, 1033. [CrossRef]

26. Feng, L.; Li, Y.; Wu, S.; Li, W.; Qiu, J.; Guo, H.; Hong, X.; Zuo, Y.; Wu, J. A review of tunable orbital angular momentum modes in fiber: Principle and generation. Appl. Sci. 2019, 9, 2408. [CrossRef]

27. Xu, Y.; Sun, J.; Frantz, J.; Shalaev, M.I.; Walasik, W.; Pandey, A.; Myers, J.D.; Bekele, R.Y.; Tsukernik, A.; Sanghera, J.S.; et al. Nonlinear metasurface for structured light with tunable orbital angular momentum. Appl. Sci. 2019, 9, 958. [CrossRef]

28. Klemes, M. Reception of OAM radio waves using pseudo-doppler interpolation techniques: A frequency-domain approach. Appl. Sci. 2019, 9, 1082. [CrossRef]

29. Li, Y.; Li, Y.; Feng, L.; Yang, C.; Li, W.; Qiu, J.; Hong, X.; Zuo, Y.; Guo, H.; Tong, W.; et al. Mode-selective photonic lanterns for orbital angular momentum mode division multiplexing. Appl. Sci. 2019, 9, 2233. [CrossRef]

30. Ruffato, G.; Massari, M.; Capaldo, P.; Romanato, F. Holographic silicon metasurfaces for total angular momentum demultiplexing applications in telecom. Appl. Sci. 2019, 9, 2387. [CrossRef]

31. Qu, Z.; Djordjevic, I.B. Orbital angular momentum multiplexed free-space optical communication systems based on coded modulation. Appl. Sci. 2018, 8, 2179. [CrossRef]

32. Lee, D.; Sasaki, H.; Fukumoto, H.; Yagi, Y.; Shimizu, T. An evaluation of orbital angular momentum multiplexing technology. Appl. Sci. 2019, 9, 1729. [CrossRef] 